\title{
Zinc transporter 3 is involved in learned fear and extinction, but not in innate fear
}

\author{
Guillaume Martel, Charles Hevi, Olivia Friebely, Trevor Baybutt, and Gleb P. Shumyatsky ${ }^{1}$ \\ Department of Genetics, Rutgers University, Piscataway, New Jersey 08854, USA
}

\begin{abstract}
Synaptically released $\mathrm{Zn}^{2+}$ is a potential modulator of neurotransmission and synaptic plasticity in fear-conditioning pathways. Zinc transporter 3 ( $\mathrm{ZnT3}$ ) knock-out (KO) mice are well suited to test the role of zinc in learned fear, because $\mathrm{ZnT3}$ is colocalized with synaptic zinc, responsible for its transport to synaptic vesicles, highly enriched in the amygdala-associated neural circuitry, and $\mathrm{ZnT3} \mathrm{KO}$ mice lack $\mathrm{Zn}^{2+}$ in synaptic vesicles. However, earlier work reported no deficiency in fear memory in ZnT3 KO mice, which is surprising based on the effects of $\mathrm{Zn}^{2+}$ on amygdala synaptic plasticity. We therefore reexamined $\mathrm{ZnT3} \mathrm{KO}$ mice in various tasks for learned and innate fear. The mutants were deficient in a weak fear-conditioning protocol using single tone-shock pairing but showed normal memory when a stronger, five-pairing protocol was used. ZnT3 KO mice were deficient in memory when a tone was presented as complex auditory information in a discontinuous fashion. Moreover, ZnT3 KO mice showed abnormality in trace fear conditioning and in fear extinction. By contrast, ZnT3 KO mice had normal anxiety. Thus, ZnT3 is involved in associative fear memory and extinction, but not in innate fear, consistent with the role of synaptic zinc in amygdala synaptic plasticity.
\end{abstract}

In the mammalian brain, the amygdala-associated neural circuitry is critical for processing innate fear and acquiring, storing, and extinguishing learned fear. Depending on the type of fear processing, the amygdala may utilize connections with the hippocampus, auditory thalamus, and auditory/perirhinal cortex, as well as the prefrontal cortex (LeDoux 2000; Milad et al. 2007). Identification of molecular markers specific to the neural circuits involved should allow for better understanding of the mechanisms underlying fear behaviors (Zirlinger et al. 2001; Shumyatsky et al. 2002). One of the molecular markers of the amygdala-associated neural circuitry is zinc transporter 3 (ZnT3). We have previously isolated the Slc30a3 gene encoding ZnT3 in the differential screening of single-cell cDNA libraries derived from individual amygdala pyramidal neurons (Shumyatsky et al. 2002). ZnT3 is one of the critical zinc transporters in the brain; it is selectively located on the vesicles of zincsecreting neurons and is responsible for most zinc released into the synapse (Palmiter et al. 1996; Wenzel et al. 1997; Linkous et al. 2008; Suh 2009). Expression pattern of the Slc30a3 gene is strikingly similar to histochemically reactive zinc (Palmiter et al. 1996; Kodirov et al. 2006; Chi et al. 2008). In neurons, histochemically reactive zinc is stored in the presynaptic vesicles that also release glutamate, which led to the hypothesis that zinc may act as a neuromodulator (Frederickson 1989). Synaptically released zinc is thought to be important for functioning of the neurotransmitters glutamate and $\gamma$-aminobutyric acid (GABA) (Qian and Noebels 2005). There is evidence for the role of synaptically released zinc in hippocampal synaptic plasticity (Li et al. 2001; Izumi et al. 2006; Qian and Noebels 2006; Huang et al. 2008). Furthermore, our earlier work indicates that zinc is involved in defining the specificity of sensory inputs in the amygdala, being critical for long-term potentiation (LTP) in the corticoamygdala pathway but not in the thalamo-amygdala pathway (Kodirov et al. 2006). In contrast to the emerging role of zinc in synaptic plasticity, its role in learning and memory has not been established. ZnT3 KO mice lack any histochemically detectable

\footnotetext{
${ }^{1}$ Corresponding author.
}

E-mail gleb@biology.rutgers.edu; fax (732) 445-1147.

Article is online at http://www.learnmem.org/cgi/doi/10.1101//m.1962010. zinc (Cole et al. 1999), providing a good model for testing the role of vesicular zinc in memory. It should be noted that earlier work has shown that ZnT3 KO mice are normal in spatial and fear learning (Cole et al. 2001).

Vesicular zinc and ZnT3 are strongly enriched in the amygdala and its afferent cortical areas as well as in the hippocampus, suggesting a role for vesicular zinc in fear and spatial memory. These anatomic observations, together with our earlier findings on the role of $\mathrm{Zn}^{2+}$ in amygdala synaptic plasticity in the rat brain (Kodirov et al. 2006), prompted us to focus our behavioral studies on memory of fear in ZnT3 KO mice. Our analysis of ZnT3 KO mice suggests that $\mathrm{ZnT3}$ is required for establishing and extinguishing memories of learned fear but is dispensable for innate fear.

\section{Results}

\section{ZnT3 and synaptically released zinc are localized in the amygdala, cortex, and hippocampus}

Using RNA in situ hybridization, we characterized the expression pattern of ZnT3 in the adult (3-mo-old) mouse brain. ZnT3 was localized in the amygdala and its afferent pathways sending the conditioned stimulus (CS) information to the amygdala. Auditory fear-related CS is delivered to the lateral (LA) and basal (BA) amygdala by two main routes: subcortical and cortical pathways. The subcortical pathway to the LA/BA is direct and originates from two thalamic areas: the medial portion $(\mathrm{MGm})$ of the medial geniculate body (MGN) and the adjacent posterior intralaminar nucleus (PIN) (LeDoux et al. 1985, 1990). Cortical projections to the LA/BA originate in secondary (TE2/TE3) auditory cortex and also in perirhinal cortex (PRh), which receives multimodal sensory inputs (Deacon et al. 1983). In addition, contextual information is delivered to the LA and BA from the hippocampus. RNA encoding ZnT3 was prominently visible in the LA, BA, and basomedial (BMA) nuclei of the amygdala, the TE3 and PRh cortical areas, the CA1 area of the hippocampus, and the dentate gyrus (DG) (Fig. 1A). While ZnT3 was strongly expressed in the cortical afferents to the amygdala, we found no expression in the MGm/PIN area of the auditory thalamus. Interestingly, ZnT3 RNA was not visible in neurons of the 

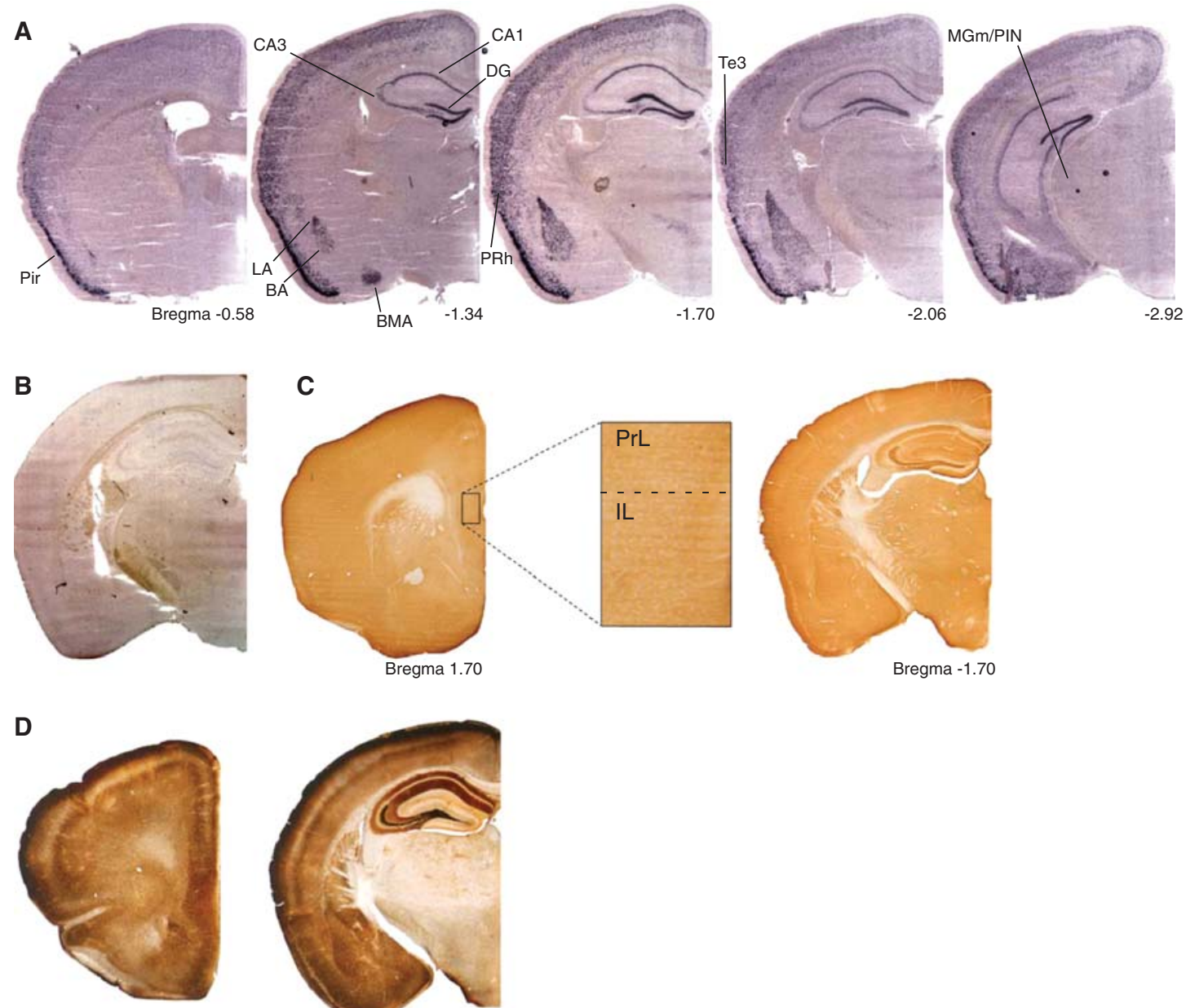

Bregma 2.00

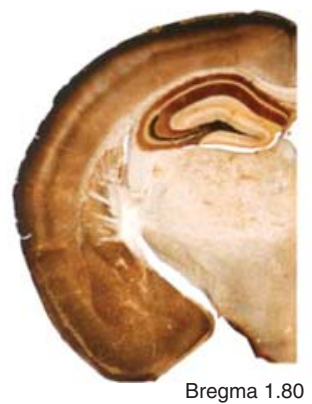

Figure 1. Expression of ZnT3 RNA and protein in the brain. (A) RNA in situ hybridization shows ZnT3 presence in the amygdala, hippocampus, TE3 area of the auditory cortex, and perirhinal cortex but not in the MGm/PIN of the auditory thalamus in the wild-type (WT) mouse brain. (B) RNA in situ hybridization shows ZnT3 absence in the brain of ZnT3 KO mice. (C) Expression pattern of ZnT3 protein detected by anti-ZnT3 antibody. Brain section on the left shows ZnT3 expression in the PFC and includes an inset showing prelimbic (PrL) and infralimbic (IL) areas. (D) Detection of synaptically released zinc by Timm's method.

prefrontal cortex (PFC), but immunocytochemistry and Timm's staining showed the presence of ZnT3 protein and synaptic zinc in the PFC. These results suggest that ZnT3 and synaptic zinc do not originate from the PFC neurons but are delivered via neuronal projections from neurons in other parts of the brain. Overall, ZnT3 expression both at the level of RNA and/or protein was almost identical to the distribution of free zinc in the brain, as analyzed by Timm's staining (Fig. 1A,C, and D).

\section{ZnT3 KO mice are deficient in a weak training protocol in fear conditioning}

Because ZnT3 is strongly expressed in the lateral and basal nuclei of the amygdala, we turned to ZnT3 KO mice to examine the consequences of the elimination of the Slc30a3 gene, which encodes ZnT3 protein, on fear memory. As expected, RNA in situ hybridization with brain tissue from ZnT3 KO mice showed no staining, confirming the absence of the Slc30a3 RNA (Fig. 1B). We first examined the mutant mice using a single tone-shock pairing for fear conditioning, which is a relatively weak training. During training, both ZnT3 KO and wild-type (WT) mice froze immediately after the shock $\left(F_{(1,20)}=16.88, P<0.001\right.$; Fig. $\left.2 \mathrm{~A}\right)$. A two-way ANOVA revealed a significant effect of genotype with ZnT3 KO mice showing a decreased level of freezing immediately after the shock $\left(F_{(1,20)}=8.45, P=0.009\right)$ as well as a significant interaction between session and genotype $\left(F_{(1,20)}=7.87, P=\right.$ $0.011)$. A Scheffe's F post-hoc analysis confirmed this effect $(P=$ 0.009), showing a significant difference between groups after the shock $(P=0.009)$, but not before the shock $(P>0.218)$. To examine long-term memory for the context, the mice were tested $24 \mathrm{~h}$ later in the same chamber (Fig. 2B). Although both genotypes established memory for the context, a Student's $t$-test revealed a significant effect of genotype with the mutants freezing significantly less than the WT mice $(t=3.2, P=0.005)$. When tested for the tone in a new context, both genotypes displayed low levels of freezing in the new context before the tone onset (Fig. 2C, pretone). Both genotypes froze at the onset of the tone (Fig. 2C, tone) compared with their freezing before the tone $\left(F_{(1,20)}=88.72, P<0.001\right)$. A two-way ANOVA showed a significant effect of genotype $\left(F_{(1,20)}=6.08, P=0.023\right)$ as well as a significant interaction between session and genotype $\left(F_{(1,20)}=\right.$ $10.43, P=0.004$ ) with the mutants freezing less than the WT controls when the tone-CS was presented. A Scheffe's F post-hoc analysis confirmed this effect showing no differences in pretone 
A

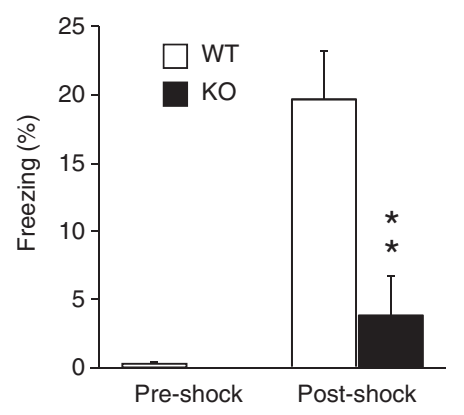

B

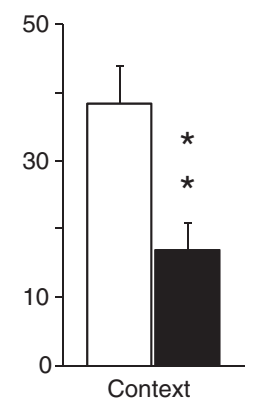

C

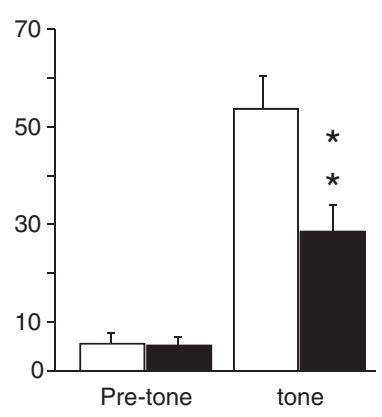

$\mathbf{F}$

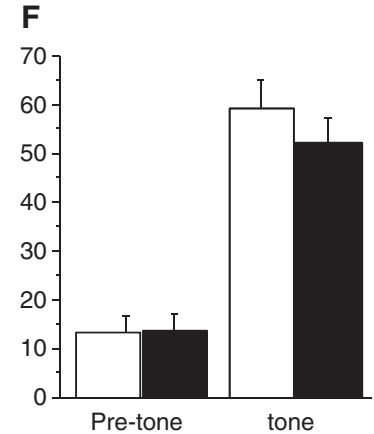

Figure 2. $\mathrm{ZnT3} \mathrm{KO}$ mice are deficient in fear conditioning in the single tone-shock-pairing protocol but are normal when five pairings are used. $(A-C)$ Results of fear conditioning in the single-pairing protocol. (A) During acquisition, ZnT3 KO mice froze significantly less than WT mice immediately after the shock. $* * P<0.01$. ( $B, C$ ) Retention data, context test, and tone test, respectively. In both tests, ZnT3 KO mice froze less than their WT littermates. ${ }^{* *} P<0.01$. ( $\left.D-F\right)$ Results of fear conditioning in the five-pairings protocol show that ZnT3 KO mice are normal during acquisition and retention for both context and tone. Results are presented as mean \pm SEM.

after the first shock (compare to postshock freezing in the single-pairing protocol) showed no differences between the genotypes either with the continuous $(P>0.930)$ or the discontinuous tone $(P>0.102)$. During testing, the mutants from both the continuous and discontinuous tone groups showed normal freezing to the context $\left(P_{s}>0.111\right.$, Fig. 3C). In the cue test, both genotypes from both groups froze at the onset of the tone $\left(P_{s}<0.001\right)$. The mice trained using the continuous tone showed no differences in freezing between the genotypes before or after tone presentation $\left(P_{s}>0.440\right.$, Fig. 3D). Therefore, the training protocol with the five $\mathrm{CS}$ unconditioned stimulus (US) pairings using a continuous ultrasound tone produces no abnormalities in the mutants. However, the group of mice that received five sequential CS-US pairings using a discontinuous ultrasound tone froze significantly less than their WT littermates. A two-way ANOVA showed a genotype effect $\left(F_{(1,17)}=7.10, P=0.016\right.$, Fig. 3D) as well as a significant interaction between session and genotype $\left(F_{(1,17)}=4.78, P=\right.$ 0.040). A Scheffe's F post-hoc analysis confirmed this effect showing a significant difference between the WT and knockout animals during freezing to the tone $(P<0.024)$. freezing $(P>0.800)$ and strong differences in freezing during tone presentation $(P<0.009)$.

We also evaluated ZnT3 KO mice in a stronger fear-conditioning protocol using five tone-shock pairings. In this experiment, we used the same tone frequency $(2.8 \mathrm{kHz})$ as well as electric shock intensity and duration as for the single-pairing protocol. During training, both genotypes learned the task $\left(F_{(6,132)}=\right.$ 42.94; $P<0.001)$ with the similar rate of progression between the genotypes $\left(P_{s}>0.363\right.$, Fig. 2D). Analysis of post-shock freezing following the first shock (compare to post-shock freezing in the single-pairing protocol) showed a significant difference between the two genotypes (Scheffe's F, $P<0.041$ ), i.e., ZnT3 KO mice froze significantly less than WT littermates. During testing, the mutants showed normal freezing to the context $(P>0.551$, Fig. 2E). Similarly, in the cue test both genotypes had the same level of freezing before and during tone presentation $\left(P_{S}>\right.$ 0.390, Fig. 2F).

\section{ZnT3 KO mice are deficient in a "complex" auditory tone protocol in fear conditioning}

We examined how the complexity of the auditory signal affects fear conditioning, by studying freezing during acquisition and retrieval for continuous vs. discontinuous tones. One group of mice was trained using a continuous ultrasound tone as the CS (WT: $n=11$, mutant: $n=9$ ); another group was trained using discontinuous ultrasound pips (WT: $n=10$; mutant: $n=9$, Fig. 3A). During training, both genotypes learned the task $\left(P_{s}<0.001\right)$ with the similar rate of progression between the genotypes in both groups $\left(P_{s}>0.096\right.$, Fig. 3B). Analysis of post-shock freezing

\section{ZnT3 KO mice are deficient in trace fear conditioning}

To probe further the contribution of $\mathrm{ZnT} 3$ to memory processes under complex training conditions, the mutants were tested in trace fear conditioning, which is thought to be dependent on the amygdala, hippocampus, and perirhinal cortex (KholodarSmith et al. 2008b). The mice were trained with five tone-shock pairings with the tone and electric shock separated by a 15 -sec gap. There was no difference in the acquisition between genotypes $\left(P_{s}>0.300 ;\right.$ Fig. $\left.4 \mathrm{~A}\right)$. Twenty-four hours later, the mice were analyzed in the training context. We found significant deficit in freezing in the $\mathrm{KO}$ animals with genotype effect $(t=2.687, P=0.014$; Fig. 4B). Three hours after the context test, the mice were examined for cued conditioning. A two-way ANOVA revealed genotype effect $\left(F_{(1,21)}=9.44, \quad P=0.006\right)$ and significant interaction $\left(F_{(2,42)}=3.96, P=0.026\right.$, Fig. $\left.4 \mathrm{C}\right)$. A Scheffe's F post-hoc analysis confirmed this effect showing a significant difference in freezing during 2 min of tone presentation $\left(P_{s}<0.005\right)$.

\section{ZnT3 KO mice are deficient in fear extinction}

We next examined ZnT3 KO mice in extinction of cued fear conditioning. During the acquisition phase, which included 10 toneshock parings, both ZnT3 KO and WT mice learned the task $\left(F_{(9,189)}=58.445 ; P<0.001\right)$ with a similar rate of progression between the genotypes in both groups $(P>0.148$, data not shown). Five hours after the training, the mice were subjected to the extinction trials with one session per day for $4 \mathrm{~d}$ (Fig. 5A). At the beginning of extinction, the mice froze $50 \%$ of the time to the tone with no significant difference between the genotype groups $(P>0.450)$. Both groups extinguished through the $4 \mathrm{~d}$ of 
A

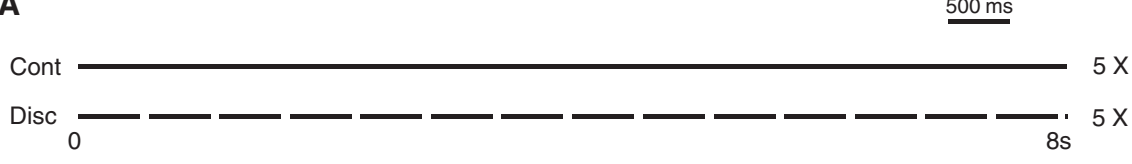

B

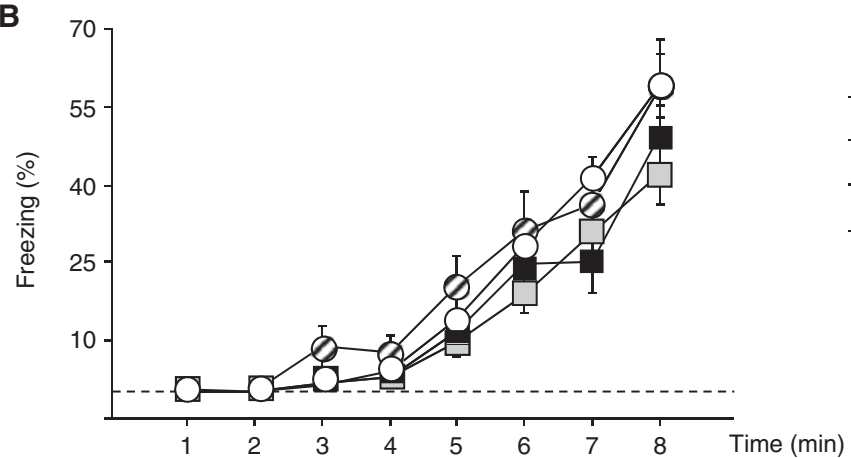

C

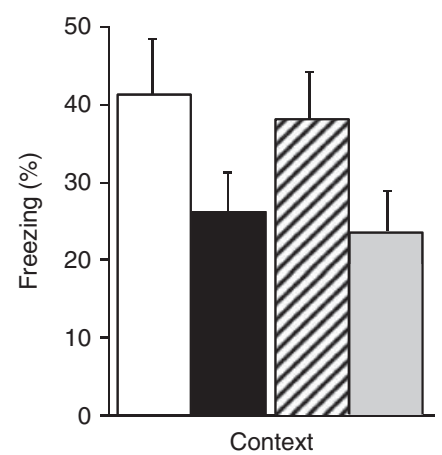

D

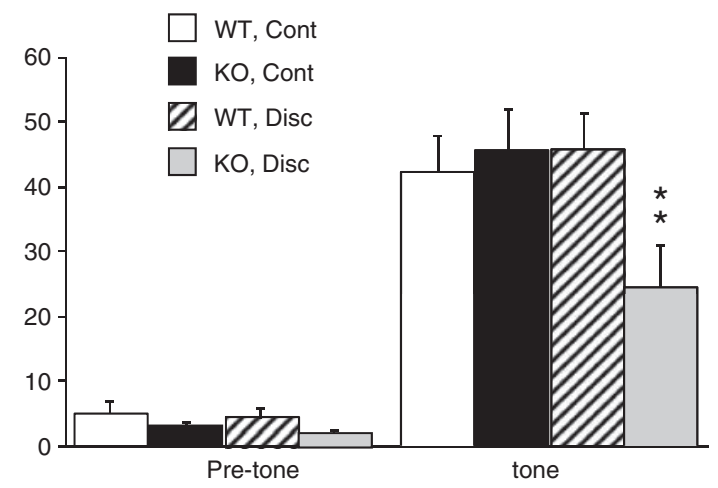

Figure 3. $\mathrm{ZnT} 3 \mathrm{KO}$ mice are normal in fear conditioning in the five-pairing protocol when a continuous tone is used as the CS, but are deficient when a discontinuous tone is used. $(A)$ Procedures for the fivepairing protocol using continuous or discontinuous tone. Both tones are at $85 \mathrm{~dB}$ and last $8 \mathrm{sec}$. For the discontinuous tone, each pulse lasted $500 \mathrm{msec}$ and the interstimulus interval was $70 \mathrm{msec}$. (B) There are no differences between the genotypes during the acquisition phase. (C) Testing for context fear conditioning showed no differences between the genotypes regardless of the type of tone used during acquisition. (D) Testing for cued fear conditioning showed normal freezing in ZnT3 KO mice for the continuous tone; however, ZnT3 KO mice froze significantly less than the WT mice for the discontinuous tone. ${ }^{*} P<0.01$. Results are presented as mean \pm SEM.

extinction $\left(F_{(19,399)}=26.09, P<0.001\right)$ to reach the level of $12 \%$ of freezing at the end of the fourth session (no difference between the groups, $P>0.370$ ). A two-way ANOVA conducted on these data revealed a difference between the genotypes $\left(F_{(1,21)}=5.27 ; P=\right.$ 0.032 ) with ZnT3 KO mice freezing less than their WT littermates. The data revealed that although both groups of mice started at the same level of freezing and reached the same level of freezing at the end of the extinction protocol, $\mathrm{ZnT} 3 \mathrm{KO}$ mice had a faster rate of extinction than their WT counterparts. Fifteen days following the last day of the extinction phase, the mice were tested in the context of acquisition to assess the initial memory (renewal). During this test both genotypes froze at a similar level (Fig. 5B). A Student's $t$-test conducted on these data confirmed the absence of difference between the genotypes $(P>0.215)$.

\section{ZnT3 KO mice are normal in pain sensitivity, innate fear, and depression-like behavior}

Is it possible that ZnT3 KO mice have fear memory deficiencies due to pain sensitivity or anxiety? To test this, ZnT3-deficient mice were first examined for pain sensitivity to the electric footshock (Fig. 6A). No difference was found between genotype groups $(P>0.640)$ in movement, vocalization, and jump in response to gradually increased levels of electric shock, which suggests that the deficits in fear conditioning were related to memory and not to sensitivity to shock.

ZnT3 mutant mice were also examined for their anxiety levels using the open field and elevated plus maze, which represent a conflict test where mice, placed in a new environment, are exploring, at the same time trying to avoid potential danger. In the elevated plus maze, mice are observed for exploring open and closed arms. Mice tend to explore new places; closed arms are perceived as less dangerous and are therefore visited more often than open arms. In the elevated plus maze the mutants spent the same amount of time in the open and closed arms compared with their WT littermates $(P>0.370$; Fig. $6 \mathrm{~B})$. In the open field ZnT3 KO mice, similar to the WT mice, showed no difference in time spent exploring the center of the arena compared with the periphery of the box $(P>0.540$; Fig. $6 \mathrm{C})$.

The mutants were also analyzed in the Porsolt's forced-swim test, which examines behavioral "despair" (Krishnan and Nestler 2008). In this test mice swim to escape from water and immobility is taken as a sign of depression. ZnT3 KO mice spent an equal amount of time not moving compared with their WT littermates, suggesting that their level of depression was normal $(P>0.100$; Fig. $6 \mathrm{D})$. These results suggest that the deficit in fear memory in ZnT3 KO mice does not result from possible abnormalities in the basal levels of pain to the electric shock, anxiety, or depression.

\section{Discussion}

The role of synaptic $\mathrm{Zn}^{2+}$ in fear learning has been unclear despite recent work showing $\mathrm{Zn}^{2+}$ involvement in synaptic plasticity in the amygdala and hippocampus, two brain regions critical for fear memory processing. To define the behavioral properties of a population of excitatory neurons that control synaptically released $\mathrm{Zn}^{2+}$ in relationship to innate and learned fear, we focused on $\mathrm{ZnT3} \mathrm{KO}$ mice. The central finding of our work is that ZnT3 is involved in subtle or complex forms of learned fear, but is not involved in innate fear.

We first characterized $\mathrm{Zn}^{2+}$ and $\mathrm{ZnT} 3$ localization in the WT mouse brain and found them to be highly enriched in the LA and BA, as well as in the cortex and hippocampus. Interestingly, $\mathrm{Zn}^{2+}$ and $\mathrm{ZnT3}$ were strongly present in the perirhinal and auditory cortical areas, but were entirely absent from the auditory thalamus, which together with the cortical pathways 
A

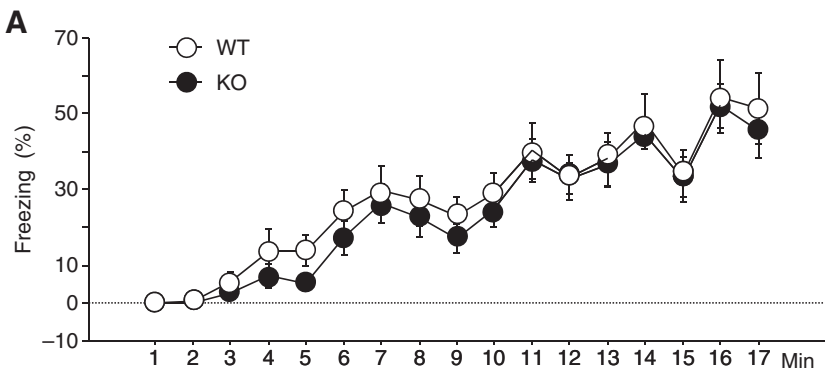

B

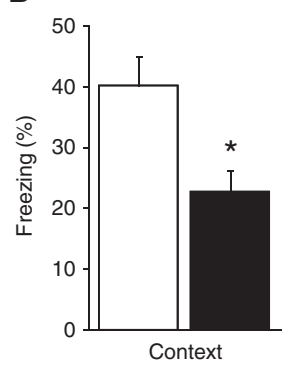

C

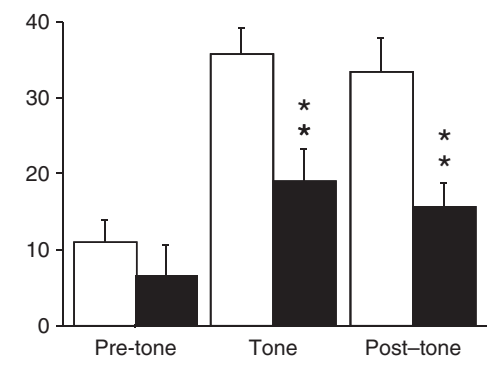

Figure 4. $\mathrm{ZnT3} \mathrm{KO}$ mice are deficient in trace fear conditioning. $(A)$ $\mathrm{ZnT3} \mathrm{KO}$ mice show normal rate of acquisition of the task. ( $B, C)$ During testing for memory for the context $(B)$ and tone $(C)$ ZnT3 KO mice froze significantly less than the control mice. ${ }^{*} P<0.05$; ${ }^{* *} P<0.01$. Results are presented as mean \pm SEM.

constitutes two major CS inputs to the LA. Thus, both the mouse and rat brains contain $\mathrm{Zn}^{2+}$ and $\mathrm{ZnT} 3$ in the cortical but not in the thalamic projections to the amygdala (Kodirov et al. 2006). Based on these immunocytochemical data and because the cortical inputs to the amygdala are thought to process more complex auditory information compared with the thalamic inputs (Armony et al. 1997; Lindquist et al. 2004), we hypothesized that ZnT3 elimination might lead to deficits in auditory processing in ZnT3 KO mice.

When subjected to a relatively weak fear-conditioning training with one presentation of the $2.8-\mathrm{kHz}$ tone-shock pairing, ZnT3 KO mice showed decreased fear memory both for tone and context. Interestingly, with more robust training using five tone-shock pairings, ZnT3 KO mice showed normal memory. During the acquisition phase in both protocols there was a postshock freezing deficit following the first tone-shock pairing. Post-shock freezing is thought to represent short-term memory for the context where the mice received the shock (Fanselow 1980); interestingly, when the mutants were tested for electric shock pain sensitivity we found no differences between the genotypes (Fig. 6A). There is also a possibility that reduced freezing immediately after the initial shock is an integral part of the initial fear response itself and most likely reflects problems in the ability of the fear circuit to encode the stimuli. To examine whether "complex" auditory information is processed differently in ZnT3 KO mice, we tested fear memory using ultrasound auditory tone $(19 \mathrm{kHz})$ presented in a continuous vs. discontinuous fashion (we used ultrasound frequency following the published protocol). During acquisition, ZnT3 KO mice showed no deficiency in postshock freezing, which is likely due to the fact that we used a 1-sec shock vs. 2 -sec shock used in the $2.8-\mathrm{kHz}$ protocols. During testing, ZnT3 KO mice froze normally to the continuous ultrasound tone but showed deficiency when the discontinuous tone was used. Although we cannot exclude that some changes in sensory discrimination exist in ZnT3 knockout mice, our experiments suggest that the frequency of the tone does not play a major role in the deficits in fear conditioning in ZnT3 KO mice. Taken together, these experiments suggest that ZnT3 is required for fear memory formation when training conditions in regard to auditory presentation are suboptimal or challenging; however, more robust and straightforward training regimens present no problem for fear memory formation. Normal fear conditioning in ZnT3 KO mice was reported by Cole et al. (2001) in the three tone-shock pairings protocol, which is similar to our results with five tone-shock pairings. However, direct comparison of the results of the two studies has to be done with caution, since we analyzed ZnT3 KO mice on the C57BL/6J background and Cole et al. (2001) examined ZnT3 KO mice on a mixed C57 $\times 129$ genetic background.

An important question is: Which parts of the circuitry are responsible for the effects we found? The deficiency in the training protocol using one shock is likely to be based on disturbances of function in the lateral nucleus of the amygdala, since $\mathrm{Zn}^{2+}$ and ZnT3 are present there at high levels. Recent work has demonstrated that the perirhinal cortex is involved in the processing of learned fear for context as well as trace conditioning, discontinuous tones, ultrasound vocalizations, and "complex auditory objects" (Campeau and Davis 1995; Corodimas and LeDoux 1995; Bucci et al. 2000; Lindquist et al. 2004; Bang et al. 2008; Kholodar-Smith et al. 2008b; Bang and Brown 2009). It is therefore possible that the deficiency in fear processing in $\mathrm{ZnT3} \mathrm{KO}$ mice, when a discontinuous tone is used, is based at least in part on improper function of the perirhinal cortex.
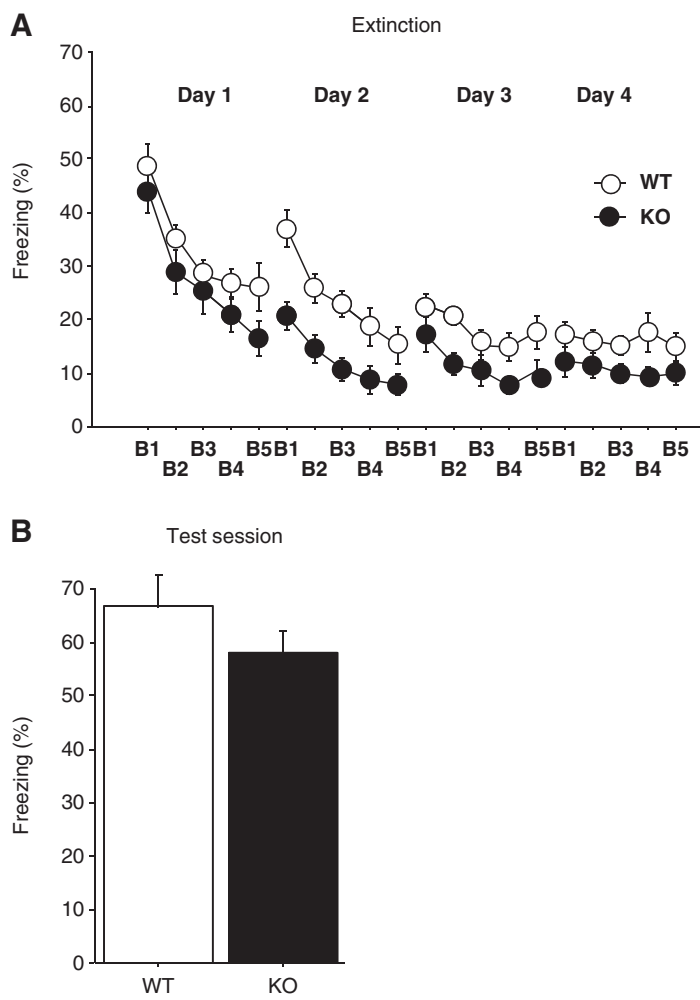

Figure 5. ZnT3 $\mathrm{KO}$ mice are deficient in extinction of fear conditioning. ZnT3 KO mice extinguished faster during cued fear conditioning. $(A)$ Percent freezing to the tone is shown by five blocks of four tone presentations during the $4 \mathrm{~d}$ of extinction. ZnT3 KO and WT mice started at the same level and reached the same level of extinction but ZnT3 KO extinguished faster than WT littermates. Results are presented as mean \pm SEM. (B) Renewal tested $15 \mathrm{~d}$ after the end of the extinction session in the context of acquisition. No difference was detected between ZnT3 and WT mice. Results are presented as mean \pm SEM. 
A
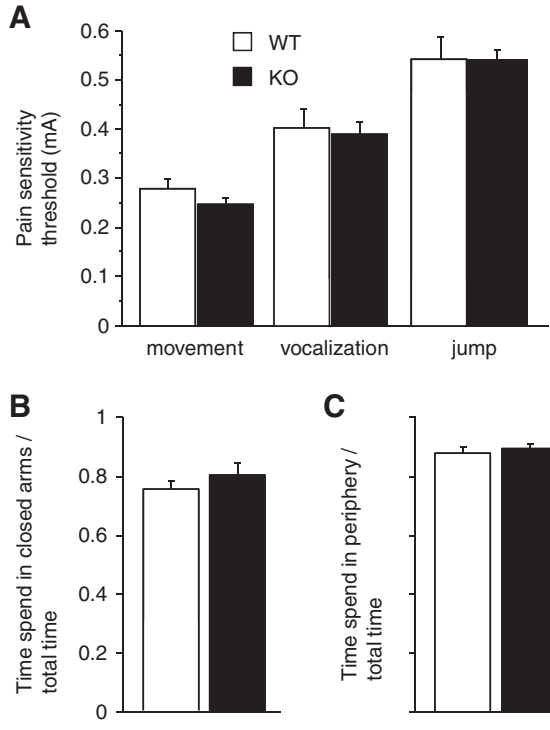

C

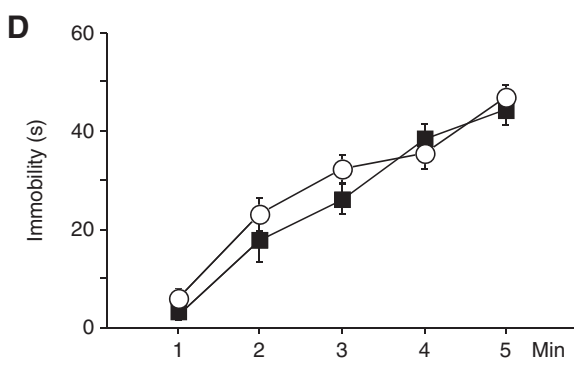

Figure 6. ZnT3 KO mice have normal shock sensitivity, innate fear, and depression-like behavior. $(A)$ No difference was found between ZnT3 KO and control mice in sensitivity to the electric shock, as measured by movement, vocalization, and jump in response to the gradual increase of the mild shock. $(B)$ The basal level of anxiety/innate fear as analyzed in the elevated plus maze is normal in ZnT3 KO mice. (C) No difference was detected between $\mathrm{ZnT3} \mathrm{KO}$ and control mice in the open field. (D) The general level of depression, as analyzed in the Porsolt's forced-swim test, is normal in ZnT3 KO mice. Results are presented as mean \pm SEM.

ZnT3 KO mice were also examined in another complex training protocol, trace fear conditioning, which requires the amygdala, hippocampus, and perirhinal cortex. We used a 15-sec time interval separating the conditioned stimulus offset (auditory tone) from the onset of the unconditioned stimulus (shock). This trace interval is thought to favor involvement of the perirhinal cortex over the hippocampus (Misane et al. 2005; KholodarSmith et al. 2008b). ZnT3 KO mice showed strong deficits in trace fear conditioning to the tone. Memory for the context was also impaired, confirming earlier reports that damage to the perirhinal cortex impairs context conditioning (Corodimas and LeDoux 1995; Bucci et al. 2000; Lindquist et al. 2004; Kholodar-Smith et al. 2008a,b).

We also found an increased rate of fear extinction in ZnT3 KO mice. Extinction is an active learning process, characterized by the development of new connections between the CS and US representations, which is thought to be dependent on the amygdala, hippocampus, and prefrontal cortex. These new connections represent inhibitory association between the CS and US, which act in parallel with the excitatory association formed during the initial CS-US learning and opposes the excitatory component (Myers and Davis 2002; Pape and Pare 2010). The balance between the inhibitory and excitatory components seems to be shifted toward stronger inhibition in ZnT3 KO mice because they extinguish faster than their WT counterparts.

To examine to what extent the deficits we observed are based on nonassociative processes, we analyzed pain sensitivity, anxiety, as well as "behavioral despair" in the mutants. In all these tests ZnT3 KO mice displayed behavior similar to that of their WT littermates. These results suggest that the deficit in fear memory in ZnT3 KO mice did not result from possible abnormalities in the basal levels of pain to the electric shock, anxiety, or depression. The absence of ZnT3 potentially might affect hearing in the mutants, because ZnT3 is present in the excitatory terminals of the dorsal cochlear nucleus (Frederickson 1989; Rubio and Juiz 1998; Cole et al. 1999). Thus, a possibility exists that our fearconditioning observations based on freezing in response to the tone might be due to hearing abnormalities. However, earlier work showed that ZnT3 KO mice have normal hearing up to a year after birth (Cole et al. 1999, 2000, 2001). As the mice used in our study were 3-5-mo old, we conclude that loss of hearing was not an issue in our fear-conditioning experiments. Is it possible that possible susceptibility to seizures leads to memory deficits in ZnT3 KO mice? Zinc deficiency is known to sensitize animals to seizure induction (Takeda et al. 2003, 2006) and it is also known that seizures disrupt consolidation processes (Moore et al. 1993; Sarkisian et al. 1997). While we cannot exclude the possibility that subseizures might occur in the mutant animals, we have never observed seizures in ZnT3 KO mice in the home cage or during any of our behavioral procedures involving electric shock in fear conditioning and testing for electric shock sensitivity. Moreover, ZnT3 KO mice showed no spontaneous seizures or acoustic- or handling-induced seizures (Cole et al. 2000). Thus, our observations and the published work of others do not support this possibility.

In agreement with our earlier paper showing that synaptically released zinc has an important role in amygdala synaptic plasticity (Kodirov et al. 2006), our present work provides behavioral evidence for the role of $\mathrm{ZnT} 3$ in associative memory processes but not in innate fear behaviors. When normal processing of fear becomes dysfunctional, various mental diseases may occur, including phobias, generalized anxiety disorder, and posttraumatic stress disorder (PTSD); some of these disorders include learned and some innate components. Understanding molecular mechanisms specific to innate or learned components will be important for designing approaches to treat fear-related mental abnormalities.

\section{Materials and Methods}

\section{Animals}

ZnT3 KO mice were generously provided by Richard Palmiter (Jackson Laboratory, Bar Harbor, ME) and backcrossed to the C57BL/6J background $(N>10)$. The targeting construct for these mice was the same as that described by Cole et al. (1999). The KO and WT mice used for these experiments were generated by breeding heterozygotes, which in turn came from the breeding of ZnT3 heterozygous mice to C57BL/6J mice (Jackson Laboratory, Bar Harbor, ME). The current approved name for this gene is Slc30a3. All mice were maintained on a 12-h light/dark cycle. Behavioral experiments were conducted during the light phase of the cycle, and mice were at least 12 wk old at the time of training. The Rutgers University Institutional Animal Care and Use Committee approved all procedures.

\section{In situ hybridization}

RNA in situ hybridization was performed as described previously (Schaeren-Wiemers and Gerfin-Moser 1993). The plasmid vector for the Slc30a3 cDNA was generously provided by Richard 
Palmiter. In short, fresh-frozen mouse brains were cut on the cryostat to obtain $20-\mu \mathrm{m}$ brain sections, which were placed on the Superfrost/Plus slides (Fisher). After fixation in freshly prepared $4 \%$ paraformaldehyde, sections were washed, treated with triethanolamine, washed, prehybridized, and hybridized with the RNA probe labeled with DIG-UTP (Roche). After overnight hybridization at $68^{\circ} \mathrm{C}$, the probe was washed and incubated with alkalinephosphatase-conjugated anti-DIG antibody (1:5000, Roche) at $4{ }^{\circ} \mathrm{C}$ overnight. After additional washes, the labeling was visualized by staining overnight with NBT/BCIP substrate (Promega).

\section{Immunocytochemistry}

Mice were perfused transcardially with saline phosphate buffer (PBS, $0.1 \mathrm{M}, \mathrm{pH} 7.4$ ) followed by $4 \%$ paraformaldehyde in PBS. After post-fixation overnight in the same fixative at $4^{\circ} \mathrm{C}$, coronal sections $(40 \mu \mathrm{m})$ were cut on a vibratome and collected in PBS. Anti-ZnT3 antibody was generously provided by Richard Palmiter (Wenzel et al. 1997).

\section{Timm's staining}

Timm's stain was performed as previously described (Kodirov et al. 2006). Mice were perfused transcardially with a buffered sulfate solution followed by a $4 \%$ paraformaldehyde. After a post-fixation for $48 \mathrm{~h}$ in the same fixative at $4^{\circ} \mathrm{C}$, brains were rinsed with phosphate-buffered saline (PBS; pH 7.3) for $30 \mathrm{~min}$. Then, coronal sections $(40 \mu \mathrm{m})$ were cut on a vibratome and collected in PBS before being put on glass slides. After drying overnight, sections were developed in the dark for $1 \mathrm{~h}$ in a developing solution and then rinsed in water. The sections were placed in 5\% sodium thiosulfate solution for $15 \mathrm{~min}$ and after three rinses in water, slides were coverslipped with glycerol and PBS mixture (1:1).

\section{Behavior}

For all behavioral tasks, mutant and control littermates (males, 3 mo old) were used. Statistical analyses used ANOVAs with genotype as the between-subject factor, and session (fear-conditioning experiment), day, area (quadrant or platform in the water maze), or zone (elevated plus maze and light-dark box) as within-subject factors. The experimenter was blind to the genotype in all studies.

\section{Fear conditioning}

In all experiments, time spent freezing was measured by the FreezeView software (Coulbourn Instruments).

\section{Single-pairing protocol}

Fear conditioning experiments were done as described (Bourtchouladze et al. 1998). On the training day, the mouse was placed in the conditioning chamber (Med Associates) for 2 min before the onset of the tone ( $30 \mathrm{sec}, 2800 \mathrm{~Hz}, 85 \mathrm{~dB})$. The last $2 \mathrm{sec}$ of the CS was paired with the continuous foot shock $(0.7 \mathrm{~mA})$. After an additional $30 \mathrm{sec}$ in the chamber, the mouse was returned to its home cage. Conditioning was assessed for 3 consecutive min in the chamber in which the mice were trained by scoring freezing behavior, which was defined as the complete lack of movement, in intervals of $5 \mathrm{sec}$. Mice (11 WT and $11 \mathrm{KO}$ mice) were tested $24 \mathrm{~h}$ after training. Testing for contextual memory occurred in the context in which mice were trained. Three hours later to test for memory for cued fear conditioning, mice were placed in a novel environment in which the tone $(120 \mathrm{sec})$ that had been presented during training was given after a 1-min habituation period (pre-CS).

\section{Five-pairings protocol}

On training day, the mouse was placed in the conditioning chamber (Med Associates) for $700 \mathrm{sec}$ during which the mouse received five pairings of the tone $(2800 \mathrm{~Hz}, 30 \mathrm{sec}, 85 \mathrm{~dB})$ and the shock $(0.7 \mathrm{~mA}, 50 \mathrm{~Hz}, 2 \mathrm{sec})$ with the intertrial interval (ITI) around 70 sec. Before the onset of the first CS-US, mice were allowed to explore the box for $2 \mathrm{~min}$. After the last tone-shock pairing, mice stayed in the box 1 min before going back to the home cage. Testing occurred $24 \mathrm{~h}$ later, first in the context in which mice were trained (contextual memory). Three hours after each contextual testing session, mice were placed in a novel environment (cued memory) in which the tone $(120 \mathrm{sec})$ that had been presented during training was given after a 1-min habituation period (pre-CS).

\section{Continuous vs. discontinuous tone protocol}

Mice were conditioned to either a continuous ( 11 WT and $9 \mathrm{KO}$ ) or discontinuous (10 WT and $9 \mathrm{KO}$ ) tone. The protocol for the discontinuous tone $(2 \mathrm{~Hz}, 14 \%$ duty cycle, each segment lasted $500 \mathrm{msec}$ and the interstimulus interval was $70 \mathrm{msec}$ ) is based on published work (Kholodar-Smith et al. 2008a). On training day, the mouse was placed in the conditioning chamber (Med Associates) for $8 \mathrm{~min}$ during which the mouse received five pairings of the tone $(19,000 \mathrm{~Hz}, 8 \mathrm{sec}, 85 \mathrm{~dB})$ and the shock $(0.7 \mathrm{~mA}, 50 \mathrm{~Hz}, 1 \mathrm{sec})$ with the ITI around $60 \mathrm{sec}$. Before the onset of the first CS-US, mice were allowed to explore the box for $2 \mathrm{~min}$. After the last tone-shock pairing, mice stayed in the box 1 min before going back to the home cage. Testing for contextual memory occurred $24 \mathrm{~h}$ later, in the context in which the mice (12 WT and $12 \mathrm{KO}$ ) were trained. To test for cued memory, $3 \mathrm{~h}$ after a contextual testing session, mice were placed in a novel environment in which the tone $(120 \mathrm{sec})$ that had been presented during training was given after a 1-min habituation period (pre-CS). The protocol for the continuous tone (five tone-shock pairings) was exactly the same as described for the discontinuous tone, except that the continuous tone was presented as an uninterrupted auditory signal.

\section{Trace fear conditioning}

After 2 min of exposure to the chamber, mice (12 WT and $11 \mathrm{KO}$ ) received five successive tone-shock presentations (tone: $30 \mathrm{sec}$ at $2800 \mathrm{~Hz}, 85 \mathrm{~dB}$; shock: $2 \mathrm{sec}, 0.7 \mathrm{~mA}$ ) with an ITI around $180 \mathrm{sec}$. The trace interval was $15 \mathrm{sec}$. After the last tone-shock pairing, mice stayed 1 additional min in the box before being returned to their home cage. Testing occurred $24 \mathrm{~h}$ later, first in the context in which the mice were trained (contextual fear conditioning). Three hours after each contextual testing session, the mice were placed in a novel environment (for testing cued fear conditioning) in which the tone $(120 \mathrm{sec})$ that has been presented during training was given after a 1 min habituation period (pre-CS). At the end of the tone, mice stayed an additional minute in the cage.

\section{Fear extinction}

During the acquisition phase, mice ( $11 \mathrm{WT}$ and $12 \mathrm{KO})$ received 10 paired presentations of CS tone (30 sec, $2.8 \mathrm{kHz}, 85 \mathrm{db})$ and US shock ( $2 \mathrm{sec}, 0.7 \mathrm{~mA}$ ) trials with an average of 75 -sec ITI. The extinction phase started $5 \mathrm{~h}$ after the acquisition in a novel context. The mice were exposed to $10 \mathrm{CS}$ tones (34-min test) with an average of 80 -sec ITI each day for 4 consecutive d. Fifteen days following the end of the fourth session of extinction the initial memory (renewal) was assessed during presentation of the tone in the acquisition context.

\section{Pain sensitivity}

Response to the electric foot shock was assessed with naïve mice (9 WT and 9 KO) as described earlier (Harrel et al. 2001; Shumyatsky et al. 2002). The intensity of the shock required to elicit running, vocalization, and jump was determined for each mouse by delivering a 1-sec-long shock every $30 \mathrm{sec}$ starting at $0.08 \mathrm{~mA}$ and increasing the shock $0.02 \mathrm{~mA}$ each time. Testing was stopped after all behaviors were noted.

\section{Open field}

Mice (12 WT and $12 \mathrm{KO}$ ) were placed individually in the corner of the open field $(43.2 \mathrm{~cm} \times 43.2 \mathrm{~cm})$ and observed for $20 \mathrm{~min}$. The 
time spent in the periphery and in the center of the arena was recorded using an automated tracking system (Open Field Activity Software, Med Associates). Results are expressed as the ratio of the time spent in the center over the total time spent in the arena.

\section{Elevated plus maze}

The elevated plus maze ( $1 \mathrm{~m}$ above the floor) consists of a center platform $(10 \mathrm{~cm} \times 10 \mathrm{~cm})$, two open arms $(40 \mathrm{~cm} \times 10 \mathrm{~cm})$, and two closed arms $(40 \mathrm{~cm} \times 10 \mathrm{~cm}$ ) within walls (height $30 \mathrm{~cm}$ ). Mice (12 WT and $12 \mathrm{KO}$ ) were placed individually in the center of the apparatus and the time spent in each arm was measured during a 5-min interval using Limelight software (Coulbourn Instruments). Results are expressed as the ratio of the time spent in closed arms over the total time spent in the maze.

\section{Forced swim test}

Mice (10 WT and $10 \mathrm{KO}$ ) were placed individually into glass cylinders (height $30 \mathrm{~cm}$, diameter $15 \mathrm{~cm}$ ) containing $15 \mathrm{~cm}$ of water, maintained at $23^{\circ} \mathrm{C}-25^{\circ} \mathrm{C}$. The total duration of immobility was measured during $5 \mathrm{~min}$. The mouse was judged to be immobile when it remained floating passively in the water.

\section{Statistical analysis}

Statistical analyses were run using Statview (SAS institute). Behavioral analyses were performed using one-way or two-way ANOVAs and Student's $t$-test. Post hoc tests were performed using Scheffe's F.

\section{Acknowledgments}

We thank Vadim Bolshakov, Louis Matzel, and Richard Palmiter for comments on the manuscript. We are grateful to the NARSAD, NIH, NSF, and Whitehall Foundation for financial support, Shoshana Vasheetz for editorial help, and A. Manzar and A. Wong for technical assistance. Research was funded in part by the New Jersey Governor's Council for Medical Research and Treatment of Autism, Special Child Health and Early Intervention Services, New Jersey Department of Health, and Senior Services.

\section{References}

Armony JL, Servan-Schreiber D, Romanski LM, Cohen JD, LeDoux JE. 1997. Stimulus generalization of fear responses: Effects of auditory cortex lesions in a computational model and in rats. Cereb Cortex 7: $157-165$.

Bang SJ, Brown TH. 2009. Perirhinal cortex supports acquired fear of auditory objects. Neurobiol Learn Mem 92: 53-62.

Bang SJ, Allen TA, Jones LK, Boguszewski P, Brown TH. 2008. Asymmetrical stimulus generalization following differential fear conditioning. Neurobiol Learn Mem 90: 200-216.

Bourtchouladze R, Abel T, Berman N, Gordon R, Lapidus K, Kandel ER. 1998. Different training procedures recruit either one or two critical periods for contextual memory consolidation, each of which requires protein synthesis and PKA. Learn Mem 5: 365-374.

Bucci DJ, Phillips RG, Burwell RD. 2000. Contributions of postrhinal and perirhinal cortex to contextual information processing. Behav Neurosci 114: $882-894$.

Campeau S, Davis M. 1995. Involvement of subcortical and cortical afferents to the lateral nucleus of the amygdala in fear conditioning measured with fear-potentiated startle in rats trained concurrently with auditory and visual conditioned stimuli. J Neurosci 15: 2312-2327.

Chi ZH, Wang X, Cai JQ, Stoltenberg M, Danscher G, Wang ZY. 2008. Zinc transporter 3 immunohistochemical tracing of sprouting mossy fibres. Neurochem Int 52: 1305-1309.

Cole TB, Wenzel HJ, Kafer KE, Schwartzkroin PA, Palmiter RD. 1999. Elimination of zinc from synaptic vesicles in the intact mouse brain by disruption of the ZnT3 gene. Proc Natl Acad Sci 96: 1716-1721.
Cole TB, Robbins CA, Wenzel HJ, Schwartzkroin PA, Palmiter RD. 2000. Seizures and neuronal damage in mice lacking vesicular zinc. Epilepsy Res 39: 153-169.

Cole TB, Martyanova A, Palmiter RD. 2001. Removing zinc from synaptic vesicles does not impair spatial learning, memory, or sensorimotor functions in the mouse. Brain Res 891: 253-265.

Corodimas KP, LeDoux JE. 1995. Disruptive effects of posttraining perirhinal cortex lesions on conditioned fear: Contributions of contextual cues. Behav Neurosci 109: 613-619.

Deacon TW, Eichenbaum H, Rosenberg P, Eckmann KW. 1983. Afferent connections of the perirhinal cortex in the rat. J Comp Neurol 220: $168-190$

Fanselow MS. 1980. Conditioned and unconditional components of post-shock freezing. Pavlov J Biol Sci 15: 177-182.

Frederickson CJ. 1989. Neurobiology of zinc and zinc-containing neurons. Int Rev Neurobiol 31: 145-238.

Harrel AV, Caldwell KK, Allan AM. 2001. Transgenic mice over-expressing the 5-HT3 receptor have enhanced learning in latent inhibition and contextual fear conditioning paradigms. In Proceedings of Society for Neuroscience 31st Annual Meeting. San Diego, CA.

Huang YZ, Pan E, Xiong ZQ, McNamara JO. 2008. Zinc-mediated transactivation of TrkB potentiates the hippocampal mossy fiber-CA3 pyramid synapse. Neuron 57: 546-558.

Izumi Y, Auberson YP, Zorumski CF. 2006. Zinc modulates bidirectional hippocampal plasticity by effects on NMDA receptors. J Neurosci 26: $7181-7188$.

Kholodar-Smith DB, Allen TA, Brown TH. 2008a. Fear conditioning to discontinuous auditory cues requires perirhinal cortical function. Behav Neurosci 122: 1178-1185.

Kholodar-Smith DB, Boguszewski P, Brown TH. 2008b. Auditory trace fear conditioning requires perirhinal cortex. Neurobiol Learn Mem 90: 537-543.

Kodirov SA, Takizawa S, Joseph J, Kandel ER, Shumyatsky GP, Bolshakov VY. 2006. Synaptically released zinc gates long-term potentiation in fear conditioning pathways. Proc Natl Acad Sci 103: 15218-15223.

Krishnan V, Nestler EJ. 2008. The molecular neurobiology of depression. Nature 455: 894-902.

LeDoux JE. 2000. Emotion circuits in the brain. Annu Rev Neurosci 23: $155-184$.

LeDoux JE, Ruggiero DA, Reis DJ. 1985. Projections to the subcortical forebrain from anatomically defined regions of the medial geniculate body in the rat. J Comp Neurol 242: 182-213.

LeDoux JE, Farb C, Ruggiero DA. 1990. Topographic organization of neurons in the acoustic thalamus that project to the amygdala. $J$ Neurosci 10: 1043-1054.

Li Y, Hough CJ, Frederickson CJ, Sarvey JM. 2001. Induction of mossy fiber $\rightarrow \mathrm{Ca} 3$ long-term potentiation requires translocation of synaptically released $\mathrm{Zn}^{2+}$. J Neurosci 21: 8015-8025.

Lindquist DH, Jarrard LE, Brown TH. 2004. Perirhinal cortex supports delay fear conditioning to rat ultrasonic social signals. J Neurosci 24: 3610-3617.

Linkous DH, Flinn JM, Koh JY, Lanzirotti A, Bertsch PM, Jones BF, Giblin LJ, Frederickson CJ. 2008. Evidence that the ZNT3 protein controls the total amount of elemental zinc in synaptic vesicles. J Histochem Cytochem 56: 3-6.

Milad MR, Wright CI, Orr SP, Pitman RK, Quirk GJ, Rauch SL. 2007. Recall of fear extinction in humans activates the ventromedial prefrontal cortex and hippocampus in concert. Biol Psychiatry 62: 446-454.

Misane I, Tovote P, Meyer M, Spiess J, Ogren SO, Stiedl O. 2005. Time-dependent involvement of the dorsal hippocampus in trace fear conditioning in mice. Hippocampus 15: 418-426.

Moore SD, Barr DS, Wilson WA. 1993. Seizure-like activity disrupts LTP in vitro. Neurosci Lett 163: 117-119.

Myers KM, Davis M. 2002. Behavioral and neural analysis of extinction. Neuron 36: $567-584$

Palmiter RD, Cole TB, Quaife CJ, Findley SD. 1996. ZnT-3, a putative transporter of zinc into synaptic vesicles. Proc Natl Acad Sci 93: 14934-14939.

Pape HC, Pare D. 2010. Plastic synaptic networks of the amygdala for the acquisition, expression, and extinction of conditioned fear. Physiol Rev 90: $419-463$

Qian J, Noebels JL. 2005. Visualization of transmitter release with zinc fluorescence detection at the mouse hippocampal mossy fibre synapse. J Physiol 566: 747-758.

Qian J, Noebels JL. 2006. Exocytosis of vesicular zinc reveals persistent depression of neurotransmitter release during metabotropic glutamate receptor long-term depression at the hippocampal CA3-CA1 synapse. J Neurosci 26: 6089-6095.

Rubio ME, Juiz JM. 1998. Chemical anatomy of excitatory endings in the dorsal cochlear nucleus of the rat: Differential synaptic distribution of aspartate aminotransferase, glutamate, and vesicular zinc. J Comp Neurol 399: 341-358. 
Sarkisian MR, Tandon P, Liu Z, Yang Y, Hori A, Holmes GL, Stafstrom CE. 1997. Multiple kainic acid seizures in the immature and adult brain: Ictal manifestations and long-term effects on learning and memory. Epilepsia 38: 1157-1166.

Schaeren-Wiemers N, Gerfin-Moser A. 1993. A single protocol to detect transcripts of various types and expression levels in neural tissue and cultured cells: In situ hybridization using digoxigenin-labelled cRNA probes. Histochemistry 100: 431-440.

Shumyatsky GP, Tsvetkov E, Malleret G, Vronskaya S, Hatton M, Hampton L, Battey JF, Dulac C, Kandel ER, Bolshakov VY. 2002.

Identification of a signaling network in lateral nucleus of amygdala important for inhibiting memory specifically related to learned fear. Cell 111: 905-918.

Suh SW. 2009. Detection of zinc translocation into apical dendrite of CA1 pyramidal neuron after electrical stimulation. J Neurosci Methods 177: $1-13$.
Takeda A, Hirate M, Tamano H, Nisibaba D, Oku N. 2003. Susceptibility to kainate-induced seizures under dietary zinc deficiency. J Neurochem $\mathbf{8 5}$ : $1575-1580$

Takeda A, Itoh H, Hirate M, Oku N. 2006. Region-specific loss of zinc in the brain in pentylentetrazole-induced seizures and seizure susceptibility in zinc deficiency. Epilepsy Res 70: 41-48.

Wenzel HJ, Cole TB, Born DE, Schwartzkroin PA, Palmiter RD. 1997. Ultrastructural localization of zinc transporter-3 (ZnT-3) to synaptic vesicle membranes within mossy fiber boutons in the hippocampus of mouse and monkey. Proc Natl Acad Sci 94: 12676-12681.

Zirlinger M, Kreiman G, Anderson DJ. 2001. Amygdala-enriched genes identified by microarray technology are restricted to specific amygdaloid subnuclei. Proc Natl Acad Sci 98: 5270-5275.

Received July 28, 2010; accepted in revised form August 31, 2010. 


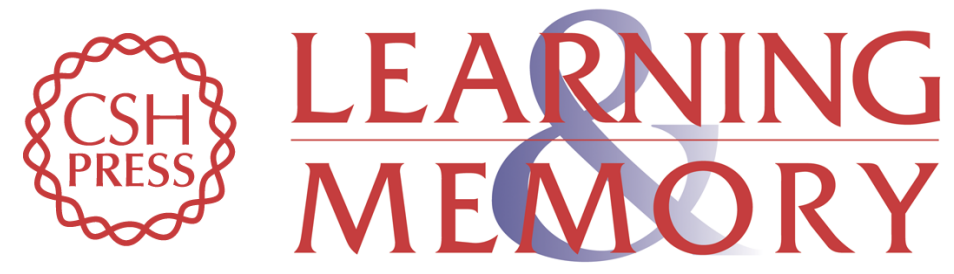

\section{Zinc transporter 3 is involved in learned fear and extinction, but not in innate fear}

Guillaume Martel, Charles Hevi, Olivia Friebely, et al.

Learn. Mem. 2010, 17:

Access the most recent version at doi:10.1101//m.1962010

References This article cites 43 articles, 12 of which can be accessed free at: http://learnmem.cshlp.org/content/17/11/582.full.html\#ref-list-1

License

Email Alerting Receive free email alerts when new articles cite this article - sign up in the box at the Service top right corner of the article or click here. 